\title{
Imaging of Pediatric Infection Within the Central Nervous System
}

\author{
Aashim Bhatia $^{1} \cdot$ Sumit Pruthi ${ }^{2}$
}

Published online: 1 September 2016

(C) Springer Science+Business Media New York 2016

\begin{abstract}
Purpose of Review Continued evolution of infectious pathogens creates constant monitoring for new infectious diseases and recurrent diseases once presumed to be eradicated. In particular, central nervous system infection in the pediatric population commonly presents in clinical practice and quick diagnosis can result in timely and appropriate treatment.

Recent Findings Declining immunizations have rejuvenated diseases in the pediatric population and multi-resistant organisms have created challenges, not only in treatment, but also in diagnosis. CNS infections require quick diagnosis and treatment to prevent life threatening complications, with imaging playing a crucial role in the workup of children.

Summary We review the imaging findings of some commonly seen infectious diseases in our pediatric population, as well as more recent appearing pathogens that have been major challenges within the medical community. A quick overview of imaging modalities commonly used in evaluation of CNS infection as well as the risk of stroke in the pediatric population secondary to infection.
\end{abstract}

Keywords Neuroimaging · Pediatrics - Central nervous system $\cdot$ Infection

This article is part of the Topical Collection on Neuroimaging.

Sumit Pruthi

sumit.pruthi@Vanderbilt.Edu

1 Monroe Carell Jr. Children's Hospital at Vanderbilt, Nashville, TN 37232, USA

2 Radiology \& Pediatrics, Pediatric Neuroradiology, Pediatric Radiology, Monroe Carell Jr. Children's Hospital at Vanderbilt, Nashville, TN 37232, USA

\section{Introduction}

Central nervous system (CNS) infection in the pediatric population commonly presents in clinical practice and is a major cause of morbidity and mortality. The spectrum of infectious diseases continues to evolve, causing challenges in diagnosis and treatment [1]. Additional factors such as declining immunizations among the pediatric population have rejuvenated diseases such as Measles [2]. Multi-resistant organisms, such as tuberculosis (TB), have also created a complex of challenges, not only in treatment, but also in diagnosis. CNS infections require quick diagnosis and treatment to prevent life threatening complications, with imaging playing a crucial role in the workup of children. While this article is not a comprehensive review of all CNS infection in the pediatric population, we review the imaging findings of some commonly seen neurological infectious diseases. A review of more recent pathogens in the pediatric population, which have been major challenges within the medical community and received a great deal of media attention will also be addressed.

\section{Modalities for Imaging CNS Infection}

\section{Ultrasound (US)}

The role of cranial US is limited in pediatric CNS infection. It is however an excellent, quick and noninvasive modality to assess ventricular size in patients with postinfectious hydrocephalus. Occasionally, findings such as intra cranial calcification, lenticulostriate vasculopathy, leptomeningeal exudate, infarcts and hemorrhagic foci can be seen. 


\section{Computed Tomography (CT)}

CT without contrast does not provide the diagnostic accuracy in rapidly diagnosing CNS infection; however, it is occasionally used in emergent setting to assess feasibility of lumbar puncture and to exclude other obvious differentials diagnoses. Addition of intravenous contrast can improve accuracy in detecting an abscess within the CNS, bony involvement with an extra-axial empyema, or leptomeningeal enhancement in suspected cases of meningitis.

\section{CT Angiography (CTA)}

Aneurysm leading to thrombotic emboli is an uncommon cause in children; however, it can be seen in the setting of mycotic aneurysms. CTA is an excellent diagnostic tool for diagnosing mycotic aneurysms, compared to MRA [3]. Similarly, CTA may be the preferred initial modality to assess for post-infectious arteritis and vasospasm, which gives the better contrast resolution over MRA for small vessel involvement. It however also results in radiation exposure and often provides only limited information of the brain parenchyma.

\section{Magnetic Resonance Imaging (MRI)}

MRI is the best diagnostic study for determining areas of involvement in the brain and spine and can improve the ability to narrow the differential to a more specific diagnosis. Diffusion weighted imaging (DWI) is the most important sequence in identifying an abscess in addition to T1 postcontrast sequences (Fig. 1) within the parenchyma, extra axial and extra cranial compartments [4]. Restriction of water molecules is visualized on DWI sequences as hyperintense signal, and correlation with the apparent diffusion coefficient (ADC) can determine if a true abscess is present. MRI allows for better diagnosis of an extra-axial empyema (Fig. 2) compared to CT and is also useful in visualizing leptomeningeal enhancement (Fig. 3). Addition of Magnetic resonance venography is extremely beneficial to assess for complications such as venous sinus thrombosis.

In children with concern for spinal cord involvement, MRI is also the best diagnostic study in defining extent of involvement, including assessment for nerve root enhancement. Additional importance of MRI is differentiating involvement of gray vs white matter within the cord, which will be discussed in further detail within the article.

\section{Vessel Wall Imaging (VWI)}

One of the common complications of CNS infection includes stroke. Possible mechanisms of underlying infection leading to stroke include promoting systemic procoagulant effects or inflammation of the wall of craniocervical vessels. For this reason, vessel wall imaging is emerging as a new diagnostic tool, which may provide the imaging findings necessary to diagnose inflammatory vessel wall changes. All major MRI manufacturers now provide vessel wall sequences. The majority are based on 3.0 T high-resolution MR imaging of vessel walls using black blood contrast $\mathrm{T} 1$ weighted sequences with fat suppression without contrast. Non-contrast MRA of the circle of Willis is also included in the evaluation. Vessel wall imaging provides information regarding vessel wall-like thickening, luminal narrowing, and enhancement.

\section{CNS Infection and Stroke}

Cerebral arteriopathy is now the major cause of AIS, with some studies demonstrating arteriopathies in up to $50-80 \%$ of cases $[5,6,7]$. The arteriopathies encompass various entities like focal cerebral arteriopathy (FCA) of childhood, arterial dissection, sickle cell anemia associated arteriopathy, and moya-moya disease, including primary and secondary forms from sickle cell, radiation, neurofibromatosis, and Down syndrome [5, 6, 7]. Recent literature suggests that infection plays a much larger role in AIS than previously documented, with varicella zoster being an established etiology [8]. The arteriopathies also appear to be important as a risk factor for recurrent AIS in children [8]. The infection leading to arteriopathies can be classified as major or mild, with major causes including encephalitis, sepsis, and meningitis, while minor infections include upper respiratory infections (URI), acute otitis media, and viral gastroenteritis. A study of non-neonatal stroke demonstrated a strong correlation of stroke with major or minor infections [9]. The risk factor of infection was more prevalent in the patient cohort than traditional pediatric risk factors such as cardiac disease and hematologic disease. The most common risk factor was an acute minor infection within 4 weeks of the stroke, with URI (33\%) being the most common cause, followed by viral syndromes (26\%) and acute otitis media (19\%). Additional studies have also documented the role of minor infection as a strong predictive risk factor for arteriopathy leading to AIS [10]. The possible mechanisms suggested by them included an inflammatory-mediated induced prothrombotic state rather than true arterial injury explaining the transient nature of the risk [11]. The vascular effects of infection in Pediatric Stroke (VIPS) Study, which is ongoing, is intended to better define the association of AIS and infection [8].

\section{Congenital Infections}

TORCH (Toxoplasma gondii, Rubella, Cytomegalovirus (CMV), and Herpesviruses) infections constitute the majority of neonatal and perinatal infections, with the more 
Fig. 1 Intracranial abscesses: 7-year-old presenting with headache and fever. Coronal T2 and postcontrast coronal $\mathrm{T} 1$ sequences (a, b) demonstrate multiple intra-axial lesions with ring enhancement. Axial DWI with ADC map (c, d) images shows intense reduced diffusion, consistent with abscesses. Microbiology of the abscesses was consistent with Streptococcus mitis and S. intermedius
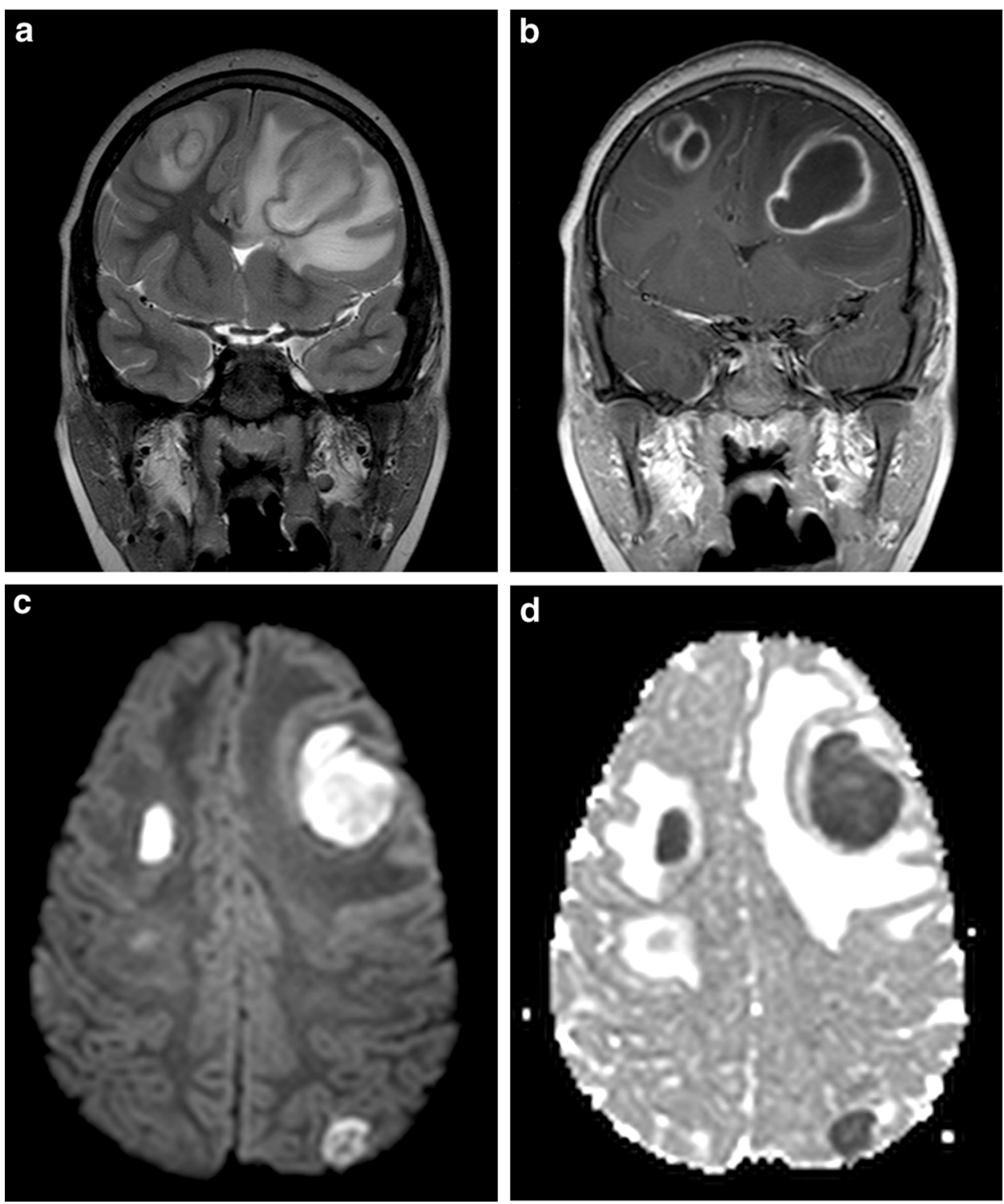

common cause of CNS infection being CMV and Herpes Simplex Virus (HSV). Imaging findings largely depend upon the age of the fetus at the time of insult, as the immune-mediated response contributing to tissue damage at later age is either absent or diminished in the fetus.

\section{Cytomegalovirus (CMV)}

CMV is one of the most common congenital/neonatal infections worldwide. Imaging findings are variable depending upon the degree of injury and timing of injury and can include intracranial calcification, ventriculomegaly, white matter disease, neuronal migrational disorders, cerebellar hypoplasia and microcephaly [12]. In the absence of calcification, CT may be normal in a large number of patients [13]. Fetal US, fetal MRI, and immediate postnatal US may be beneficial in early diagnosis [14]. MRI is the best study in evaluating extent of disease and can provide prognostic findings for development in the child [12].

Calcifications are typically periventricular with a thick and chunky appearance (Fig. 4). This is the most common imaging finding in congenital CMV [12] with infection during the first trimester. Additional areas commonly involved include the basal ganglia and brain parenchyma, which are usually faint and punctate [12]. The migrational disorders can vary from a spectrum of lissencephaly to pachygyria, to diffuse or focal polymicrogyria [12] with advancing fetal age at the time of infection. Imaging findings commonly encountered in late third trimester or asymptomatic infections include anterior temporal 

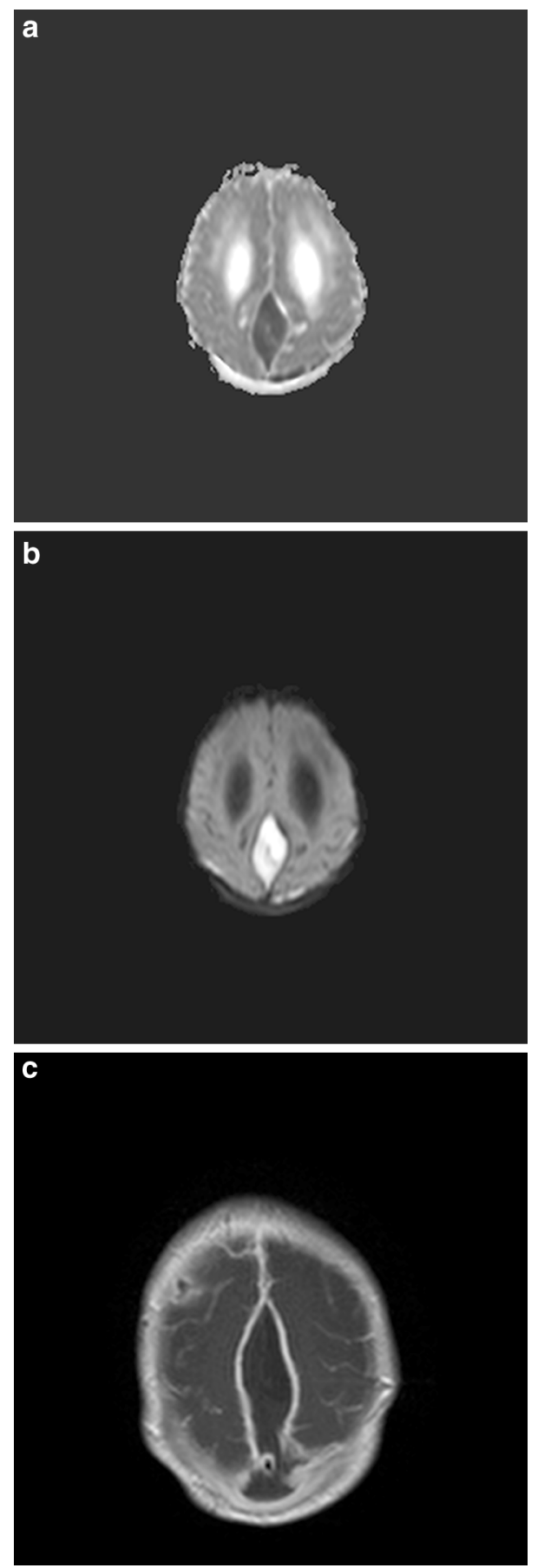

Fig. 2 Subdural empyema: 3-month-old presenting with fever. Axial DWI with ADC map (a, b) and post contrast axial T1 (c) images demonstrate a large rim enhancing collection along the interhemispheric region with reduced diffusion, consistent with a subdural empyema

cysts/cystic change and white matter abnormalities [12]. White matter abnormality is usually multifocal with bilateral posterior predominant pattern. In the authors opinion, a combination of findings of anterior temporal cysts, migrational abnormalities, scattered white matter

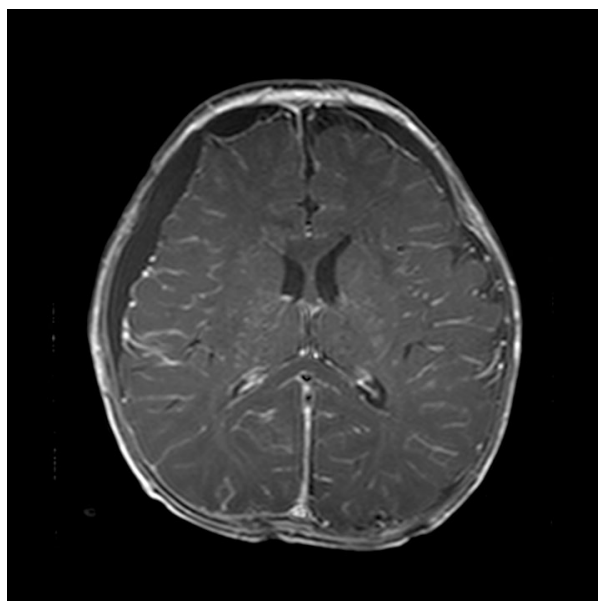

Fig. 3 Strep meningitis: 2-year-old with Streptococcus meningitis with diffuse leptomeningeal enhancement on postcontrast $\mathrm{T} 1-\mathrm{W}$ images

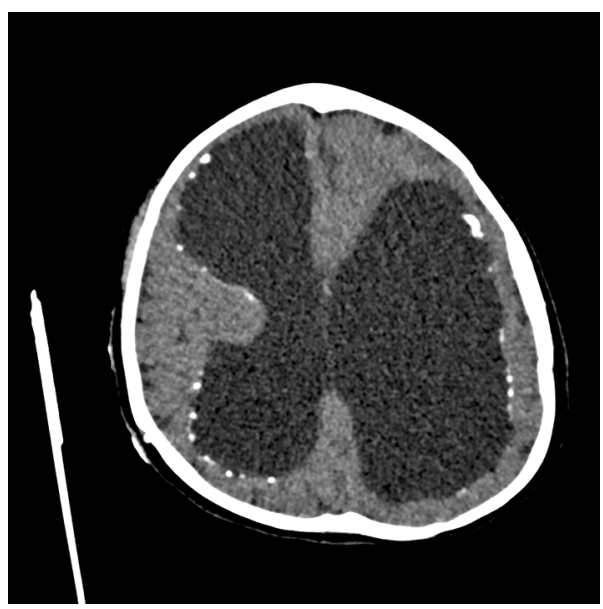

Fig. 4 CMV 17-year-old with perintala history of CMV. CT demonstrates periventricular calcifications with enlarged lateral ventricles secondary to perinatal hydrocephalus realted to CMV

hyperintensities, and clinical evidence of sensorineural hearing loss is virtually diagnostic of congenital CMV infection.

\section{Lymphocytic Choriomeningitis Virus}

Lymphocytic choriomeningitis virus, belonging to the arenaviridae family, can lead to both acquired and congenital forms of CNS infection. Congenital infection, usually acquired transplacentally has strong tropism for the brain. Imaging findings significantly overlap with congenital CMV and Toxoplasmosis infections and include microcephaly, periventricular calcifications, hydrocephalus, cerebellar hypoplasia, and migrational abnormalities. Although the exact incidence of the infection is unknown, it is important to consider LCMV infection as a differential to CMV infection [15]. 


\section{Pseudo-TORCH Syndrome}

Imaging findings of diffuse and scattered intracranial calcification, particularly periventricular in a neonate or young child is often presumed to be from TORCH infections. It is important to note that other genetic disorders such as Pseudo-Torch syndrome and Aicardi-Goutieres syndrome (AGS) can mimic congenital infections. PseudoTorch syndrome correctly knows as Band-like calcification with simplified gyration and polymicrogyria is an autosomal recessive disorder [16]. Some consider AGS and pseudo-torch syndrome as similar entities at different spectrums.

\section{Herpesviruses}

HSV infection in neonates is suggested in $\sim 1$ out of 3200 deliveries, commonly acquired during delivery, which is not limited to vaginal delivery and can be acquired with caesarian delivery $[17,18]$. HSV-2 constitutes $70-80 \%$ of neonatal infections with more morbidity and mortality compared to adult form caused by HSV1. Diffuse bilateral abnormalities were common findings seen in a retrospective analysis of 29 patients, with the thalami being the most common anatomical structure involved [18]. Unlike adults, where temporal lobe involvement is a common finding with sparing of deep gray nuclei, neonatal infection is a more diffuse process suggesting the developing brain is more susceptible to HSV infection. Studies have demonstrated DWI as an important sequence in depicting early CNS involvement, with follow-up imaging demonstrating cystic encephalomalacia (Fig. 5) in the regions of reduced diffusion [18-20]. Other rare findings in HSV-2 have been described in case reports, with significant brainstem involvement, best visualized on DWI [21].

\section{Zika Virus}

There has been a recent outbreak of Zika virus in Latin America, with the greatest number of cases in Brazil as of May 2015, with confirmed reports of the disease from 23 different countries. Zika virus is a mosquito-borne disease, related to Dengue, Yellow fever, and West Nile Virus, spread by the Aedes genus, with the major vector mosquito being the Aedes Aegypti, most commonly found in the southern part of the Unites States. Zika is caused by a RNA virus which is presumed to cause congenital infections acquired during pregnancy. It was first discovered in Uganda in 1947 and is common in Africa and Asia. There also has been reported cases of sexual transmission.

The most striking fetal abnormality thought to be linked to Zika virus includes microcephaly [22•]. While the imaging data is limited, multiple brain abnormalities have
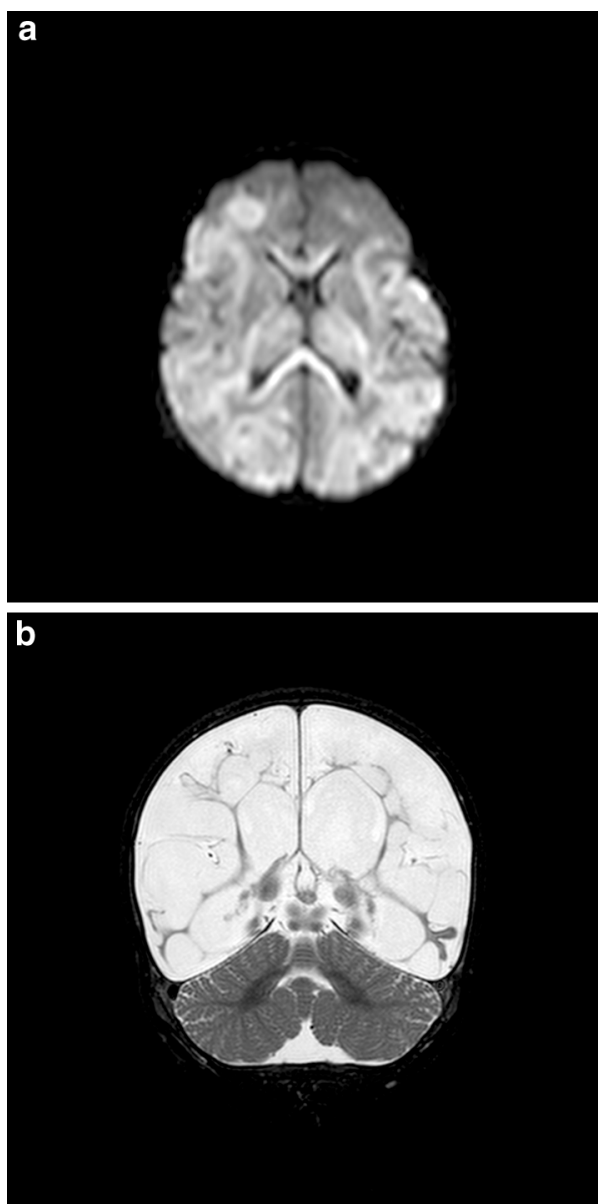

Fig. 5 HSV: 1-year-old with congenital HSV infection, with DWI (a) demonstrating restricted diffusion throughout the cerebral hemispheres. Year later the areas of reduced diffusion now demonstrate severe cystic encephalomalacia on coronal T2-W image (b)

been described: intracranial calcifications, ventriculomegaly, and neuronal migration disorders (lissencephaly and pachygyria), findings very similar to congenital CMV. Other anomalies described include congenital contractures and clubfoot as well as a spectrum of ocular abnormalities [23]. The evidence regarding a causal link between Zika virus infections during pregnancy and congenital CNS malformations is growing, although the available information is not yet sufficient to confirm it [24]. There has not been a pathogen to cause such severe congenital abnormalities during fetal development in the past few decades, possibly making the new mnemonic for congenital infections ZTORCH (Zika, Other such as HIV, Varicella, Syphilis, Toxoplasmosis, Rubella, Cytomegalovirus, Herpes).

In addition, Zika virus has been associated with Guillain-Barre Syndrome. An outbreak occurred in French Polynesia in 2013, a population of 270,000 , with 42 reported cases [24]. In January 2016, El Salvador reported a 3 fold increase in the number of cases of Guillain-Barre Syndrome. 


\section{Viral}

\section{Enterovirus D68}

Over the past few years, Enterovirus D68 or suspected Enterovirus D68 cases have been of great concern in the medical community [25, 26•]. From August 2014 to July 2015, the Center of Disease Control (CDC) has collected 120 pediatric cases of polio-like presentation across the United States with acute flaccid paralysis and MRI findings with spinal cord abnormalities, that predominantly involve the gray matter [27]. A majority of the patients have not completely recovered, with $1 / 3$ showing no improvement at the time of this article construction and there is no effective treatment at this time.

In our experience, which correlates with other studies, axial T2 MRI best demonstrated the hyperintense signal, which predominantly involved gray matter (Fig. 6), extending over multiple vertebral levels [26•, 28, 29]. The signal abnormality primarily involves the cervical region (Fig. 7) usually without associated enhancement or reduced diffusion. Additional studies have demonstrated abnormalities within the brainstem, however, without supratentorial lesions [29]. In our experience, follow-up imaging within a limited patient population showed improvement without complete resolution and a few demonstrated complete resolution. Additional findings of nerve root enhancement were seen later in the course of disease process [29].

While these patients had a suspected cause of Enterovirus D68 based on nasopharyngeal secretions and correlating with the outbreaks of respiratory related disease, CSF samples were not consistently positive in the patient population. It remains unclear whether this cluster of cases is a cause of Enterovirus D68 or other possible pathogens. Part of the inconsistency in the CSF samples may be partially due to the difficulty in isolating Enterovirus and possibly the timing of CSF collection during the disease

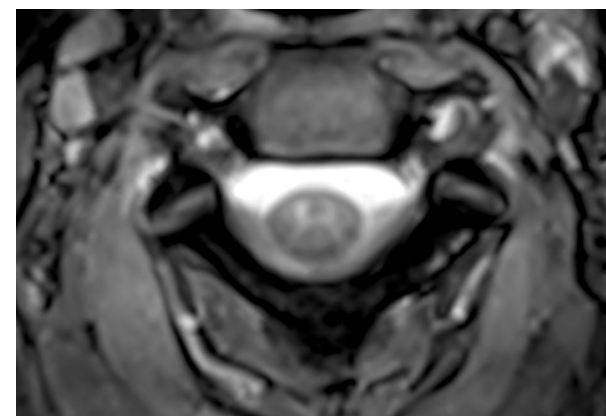

Fig. 6 Enterovirus D68: 7-year-old with recent viral infection and lower extremity weakness, lab work suspected for Enterovirus D68. Axial T2 demonstrates T2 hyperintense signal within the cervical cord, primarily involving the gray matter course. It is important to note that these cases present as other viral causes of motor neuron dominant flaccid paralysis, such as poliovirus and Enterovirus A71 encephalomyelitis [26•, 30-32].

\section{Measles}

While Measles has been a rare disease over the past two decades and according to the CDC, eradicated in 2000, it has made a resurgence, primarily due to increased number of unvaccinated children [2]. The CDC reported 178 cases from January to March 2015, with the largest increased rate of cases in 2014. Given the trend, it is paramount that clinicians are aware of the clinical presentation and radiologists are aware of the imaging findings [33]. Measles presents within the CNS in three different pathways, acute postinfectious encephalitis, acute progressive encephalitis, and subacute sclerosing panencephalitis.
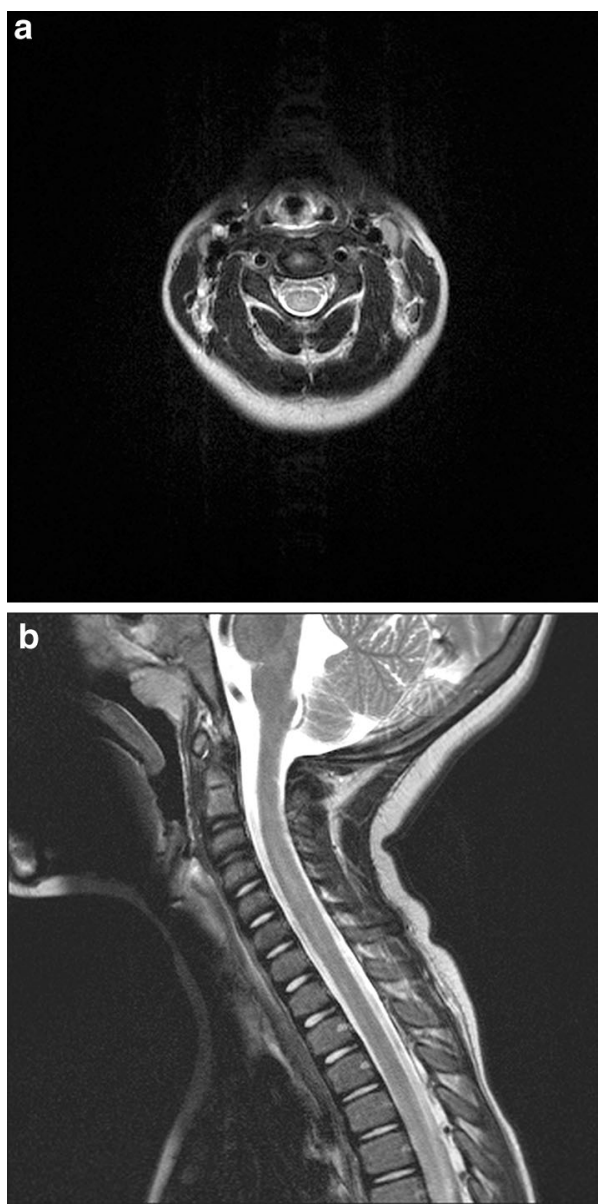

Fig. 7 Enterovirus D68 7-year-old with recent viral infection, fever, and upper extremity paralysis, lab work suspected for Enterovirus D68. Axial and sagittal T2-W images $(\mathbf{a}, \mathbf{b})$ demonstrates $\mathrm{T} 2$ hyperintense signal within the cervical cord spanning multiple levels, primarily involving the gray matter 
A review of six pediatric patients with acute Measles encephalitis demonstrated abnormal T2 hyperintensity with widely distributed, multifocal involvement in both cerebral hemispheres with swelling of the cortex [34]. There was also bilateral, symmetrical involvement of the putamen and caudate nucleus, with associated restricted diffusion. Three of the patients demonstrated subacute gyriform hemorrhage and asymmetrical gyriform enhancement.

With Measles is still more common elsewhere in the world, subacute sclerosing panencephalitis (SSPE), a postinfectious neurologic complication of measles, is another manifestation that can present itself in the United States. In particular children who traveled overseas at a young age and adopted children. Diagnosis is usually made based on typical clinical features, EEG findings and abnormal measles titers in CSF. Neuroimaging findings usually include abnormal T2 hyperintense areas predominantly involving the parieto-occipital periventricular and subcortical white matter in the earlier stages progressing to diffuse cerebral and cerebellar atrophy.

\section{Influenza-Associated Encephalitis/Encephalopathy (IAEE)}

IAEE is characterized by fever, convulsion, and neurological deficit. The imaging findings consist of symmetric intraparenchymal lesions including the thalami, periventricular white matter, brain stem, and cerebellum [35]. The literature has also reported a reversible lesion in the splenium of the corpus callosum with reduced diffusion, Acute myelopathy of the spine may develop, with imaging depicting a single lesion extending over 2 vertebral segments with mild enhancement [36]. The lesions within the spine are also reversible, with the differential including rotaivirus, measles, and mumps.

\section{Arthropod-Borne Viruses}

Arthropod-borne encephalitis viruses represent a significant public health challenge. While belonging to multiple different families of viruses, the infection usually spreads from a bite of an infected arthropod, usually a mosquito or tick lending them the name of Arboviruses. The more common viral infections within this group encountered in North America include the following: West Nile, California encephalitis group, and St. Louis encephalitis. Diagnosis of almost all arbovirus infections is most commonly based on serum and/or CSF serology. MRI findings are often nonspecific in viral encephalitis and have similar imaging findings, including diffusely scattered or confluent areas of T2 hyperintensity with variable mass effect and edema [1]. There maybe associated enhancement, with post $\mathrm{T} 1$ post-gadolinium sequences showing diffuse enhancement with leptomeningeal involvement $[1,37]$.

\section{Human Immunodeficiency Virus (HIV)/Acquired Immune Deficiency Syndrome (AIDS)}

Children with HIV/AIDS encephalopathy without antiretroviral therapy can have severe neurological deterioration with significant imaging findings. The most common locations of high prevalence include the countries of sub-Saharan Africa. In the untreated pediatric population, prevalence of HIV encephalopathy (HIVE) has ranged from 20 to $60 \%$. HIVE is brain damage and malfunction secondary to HIV-1. The most common imaging findings include global atrophy, white matter signal change with volume loss, and thinning of the corpus callosum [38-40].

More advanced imaging such as Diffusion Tensor Imaging (DTI) has shown a decrease in structural connectivity in HIV patients versus controls [41•]. Cortical gray matter thickness has also been measured with MRI and shows a thinner cortex in the sensorimotor regions of the brain as well as frontal lobe motor regions [41•]. The various modalities and MRI tools have shown that overall volume loss in HIVE is the end result if untreated. With antiretroviral therapy, this is no longer commonly seen in North America.

\section{Bacteria}

\section{Tuberculosis}

Tuberculosis (TB) has remained a common infectious pathogen throughout the world, secondary to acquired immunodeficiency syndrome (AIDS) and multi-drug-resistant TB. $60-70 \%$ of cases are below the age of 20 and is another reason pediatric radiologists and neuroradiologists should be familiar with the imaging findings [42]. Imaging findings within the CNS are widespread and some of the classical findings will be discussed.

Tuberculous meningitis is the most common manifestation within the CNS. The typical findings include basal meningeal enhancement, hydrocephalus, and supratentorial and brainstem infarctions [42, 43]. The leptomeningeal enhancement is typically diffuse involvement of the basal subarachnoid cisterns, as well as involvement over the cerebral convexities, the sylvian fissures, and the tentorium. Hydrocephalus is also common, particularly in the pediatric population, which is secondary to obstruction of CSF absorption in subarachnoid spaces secondary to TB exudate, which would be a communicating type of hydrocephalus. Infarctions secondary to basal meningitis and the exudate lead to panarteritis with secondary thrombosis and occlusion [42, 43]. 
Parenchymal tuberculomas usually appear infratentorially in children, however, not limited to this region $[42,43]$. Other regions of involvement include the supratentorial brain (Fig. 8), intrasellar region, brain stem, thalami, basal ganglia, pineal region, and the ventricles. The non-caseating granuloma is usually slightly hypodense on CT with homogenous enhancement of the solid components. The lesions on MRI are T1 hypointense and T2 hyperintense, with homogenous enhancement. Studies have demonstrated in the early stage of these lesions that contiguous vasogenic edema may surround the lesion within the white matter. Solid caseating granulomas demonstrate heterogeneous enhancement in the central portion, whereas the capsule has ring enhancement. Once the tuberculoma proceeds to central liquefaction of caseous material, there is a hypodense core with peripheral enhancement on CT. Central MRI characteristics are similar to the non-caseating stage, with T1 hypointensity and T2 hyperintensity. However, postcontrast demonstrates prominent peripheral enhancement of the lesion. DWI can help distinguish a tuberculoma from a tuberculous abscess, with the abscess demonstrating central reduced diffusion. Additional findings to suggest an abscess include larger in size and multiloculated [4, 42, 43].

With involvement of the spine, there can be linear or nodular enhancement along the nerve roots with obliteration of the subarachnoid space [42, 43]. Syringomyelia is also a complication of TB, following CSF signal on all sequences and without enhancement.

\section{Fungal}

Fungal involvement of the CNS is most commonly seen in immunocompromised children. Imaging characteristics on MRI can be generalized as heterogeneous reduced diffusion or ringlike reduced diffusion with the thin areas of peripheral enhancement [44]. Bacterial abscesses tend to have more homogenous reduced diffusion centrally.

\section{Coccidioidomycosis}

The differential diagnosis of TB meningitis includes coccidioidomycosis and cryptococcosis [42]. Cocciodomycosis is more common in the southwest with pulmonary
Fig. 8 TB: 28-month-old male with headaches and recent exposure to family member visiting from Mexico. Axial T2 and postcontrast $\mathrm{T} 1$ sequences $(\mathbf{a}, \mathbf{b}, \mathbf{c})$ demonstrate a large tuberculoma centered in the left thalamus secondary to $\mathrm{TB}$ meningitis with basal leptomeningeal enhancement. The tuberculoma is $\mathrm{T} 2$ hypointense and has ring enhancement. DWI sequence (d) shows multiple associated lenticulostriate infarcts and lack of restricted diffusion confirming a tuberculoma as opposed to an abscess
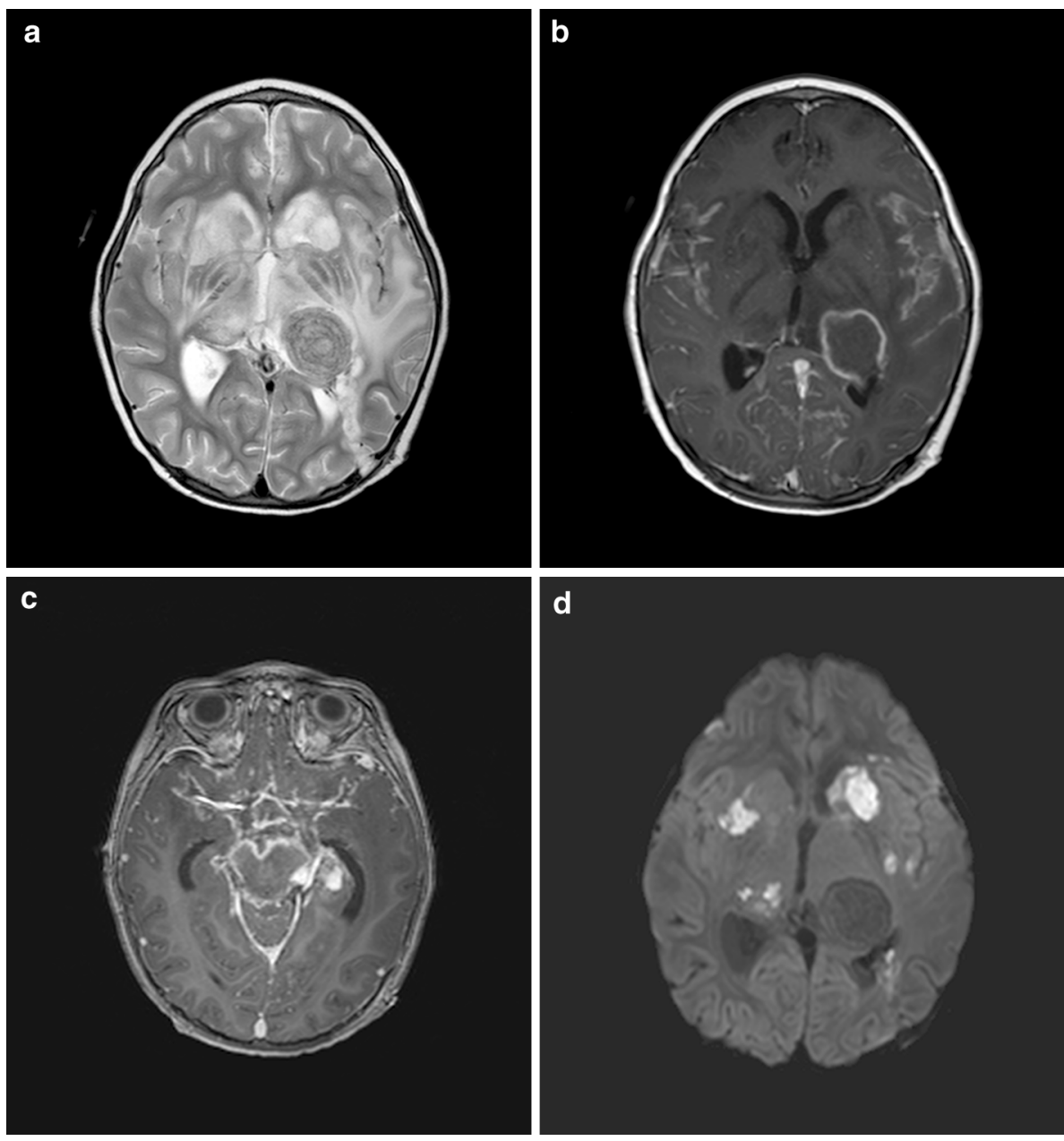
Fig. 9 Cryptococcosis:

36-year-old with Axial T2

sequence (a) showing multiple

hyperintense punctate lesions within the ganglia consistent with gelatinous pseudocysts. DWI (b) correlating with the areas of signal abnormality as restrcited diffuison
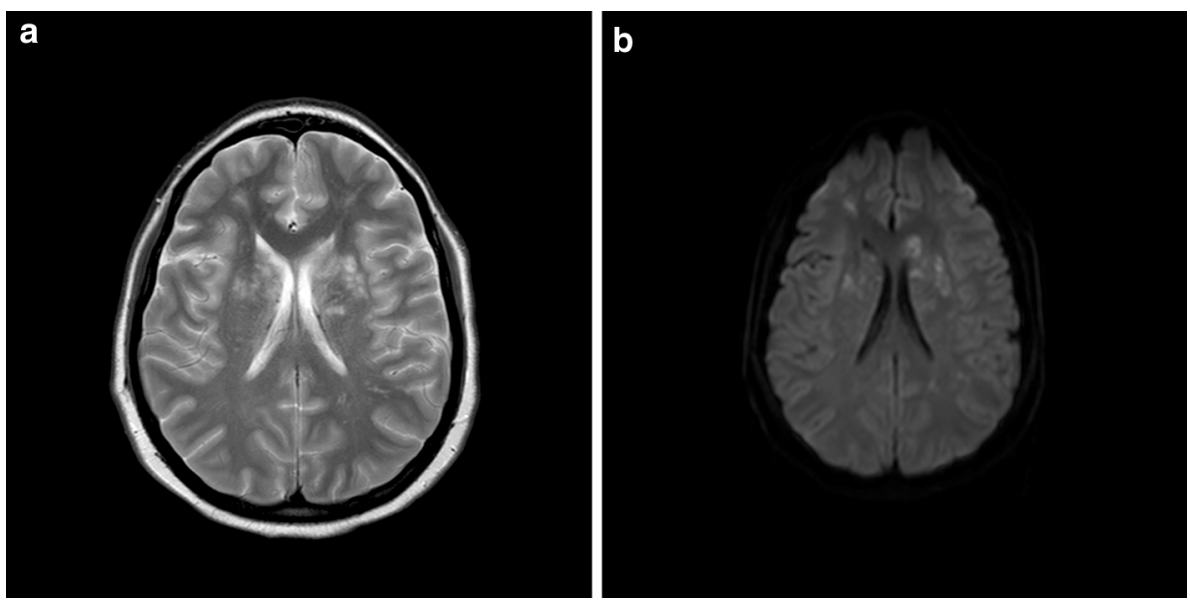

disease the most common presentation. While rare, CNS involvement can have basal meningeal enhancement. CNS involvement of coccidioidomycosis is secondary to lymphohematogenous spread from primary infection in the lungs. Common imaging findings include hydrocephalus, cerebral infarction, vasculitis, abscesses, and spinal arachnoiditis. Communicating hydrocephalus is secondary to obstruction of arachnoid granulations. Spinal cord involvement can extend inferiority along the cord as leptomeningeal enhancement. Vertebral involvement can be seen, leading to epidural extension, with a study demonstrating CNS involvement in 5 of 39 patients with vertebral involvement [44].

\section{Cryptococcosis}

Cryptococcosis is a common fungal infection in the CNS, however, rare in children with most cases seen in adults as demonstrated. Similar imaging findings to coccidioidomycosis include basal meningeal enhancement. Gelatinous pseudocysts may develop in the basal ganglia (Fig. 9), which is an important diagnostic clue as well as hydrocephalus. Elatinous pseudocysts appear T2 hyperintense with a hypointense ring likely representing methemoglobin blood products in the capsule wall or activated macrophages producing free radicals and paramagnetic susceptibility artifact. There is no post contrast enhancement seen and the lesion may be associated with restricted diffusion [44].

\section{Conclusion}

In general, early diagnosis in fetal development and neonates is best performed with ultrasound, due to lack of radiation and ability to perform without sedation, also repeat imaging can be performed without concern. MRI provides the greatest detail of brain development, injury, and findings not easily seen on CT or US, such as migrational disorders.
Evaluation of myelitis of the spine is demonstrated on MRI with the ability to locate areas of involvement to gray or white matter, which is important in diagnosis of cases such as Enterovirus D68 $\mathrm{m}$ which has a predilection to involve the gray matter. It still remains unclear if the cases of flaccid paralysis that appeared during the 2014 winter season, the same time of increased cases of respiratory disease secondary to Enterovirus D68, are truly related. The CDC collected cases from across the United States and the findings suggest a polio-like viral infection, with involvement of the anterior horns of the gray matter, demonstrated as $\mathrm{T} 2$ hyperintensity. The clinical presentation and MRI findings together were cases submitted to the CDC and radiologists and clinicians should be aware of these findings, which may help determine the suspected viral pathogen and then allow for vaccination production.

The associated risk of infection and stroke is of great interest. The majority of pediatric stroke cases are idiopathic, and multiple studies have recently demonstrated the association of even mild infection leading to stroke, which may now explain multiple cases of stroke without an etiology. The VIPS study is ongoing and will further elaborate on the associated risk of infection with stroke. It is important for radiologists to offer the most reliable diagnostic testing in cases of suspected stroke, with MRI including nonconventional sequences such as DWI, to best evaluate CNS involvement. More advanced imaging, including vessel wall imaging with MRI show promise and can demonstrate involvement of intracranial arterial vasculature, predisposing to strokes.

Some of the infectious pathogens discussed are still common today, with diseases such as congenital/neonantal CMV still a cause of serious complications during fetal and neonatal development. Radiologists should be aware of the findings in fetal MRI or fetal US, which can help guide parents. Early findings also provide prognostic information, which can be beneficial to the medical team and allow parents know what to expect for their child's development. 
Other diseases such as Measles once thought to be eradicated have now once again become a player in the world of infectious diseases. Outbreaks across the country have been reported and if not diagnosed early can be lethal in children. In addition, Measles being highly contagious can result in larger outbreaks if not diagnosed and treated, necessitating radiologists to be familiar with this disease, imaging findings, and multiple pathways of CNS involvement.

This article serves an update to radiologists and clinicians on common infectious diseases in the CNS and common imaging findings, while there are many other pathogens, some are more recent that textbooks have not discussed.

\section{Compliance with Ethical Standards}

Conflict of Interest Aashim Bhatia and Sumit Pruthi each declare no potential conflicts of interest.

Human and Animal Rights and Informed Consent This article does not contain any studies with human or animal subjects performed by any of the authors.

\section{References}

Papers of particular interest, published recently, have been highlighted as:

- Of importance

1. Silvia MT, Licht DJ. Pediatric central nervous system infections and inflammatory white matter disease. Pediatr Clin North Am. 2005;52(4):1107-26. doi:10.1016/j.pcl.2005.03.003.

2. Salmon DA, Dudley MZ, Glanz JM, Omer SB. Vaccine hesitancy causes, consequences, and a call to action. Am J Prev Med. 2015;49(Fall):S391-8. doi:10.1016/j.amepre.2015.06.009.

3. Lee W-K, Mossop PJ, Little AF, et al. Infected (mycotic) aneurysms: spectrum of imaging appearances and management. Radiographics. 2008;28(7):1853-68. doi:10.1148/rg.287085054.

4. Bonfield CM, Sharma J, Dobson S. Pediatric intracranial abscesses. J Infect. 2015;71:10-4. doi:10.1016/j.jinf.2015.04.012.

5. Wintermark M, Hills NK, DeVeber GA, et al. Arteriopathy diagnosis in childhood arterial ischemic stroke: results of the vascular effects of infection in pediatric stroke study. Stroke. 2014;45(12):3597-605. doi:10.1161/STROKEAHA.114.007404.

6. Ganesan V, Prengler M, McShane MA, Wade AM, Kirkham FJ. Investigation of risk factors in children with arterial ischemic stroke. Ann Neurol. 2003;53(2):167-73. doi:10.1002/ana.10423.

7. Amlie-Lefond C, Bernard TJ, Sebire G, et al. Predictors of cerebral arteriopathy in children with arterial ischemic stroke; results of the international pediatric stroke study. Circulation. 2009;119(10):1417-23. doi:10.1161/CIRCULATIONA HA.108.806307.

8. Glaser C, Glidden D, Hills NK, Leiva-salinas C, Wintermark M, Gabrielle A. The vascular effects of infection in pediatric stroke (VIPS) study. J Child Neurol. 2012;26(9):1101-10. doi:10.1177/ 0883073811408089.The.

9. Hills NK, Johnston SC, Sidney S, Zielinski BA, Fullerton HJ. Recent trauma and acute infection as risk factors for childhood arterial ischemic stroke. Ann Neurol. 2012;72(6):850-8. doi:10. 1002/ana.23688.

10. Amlie-Lefond C, Bernard TJ, Sébire G, et al. Predictors of cerebral arteriopathy in children with arterial ischemic stroke: results of the international pediatric stroke study. Circulation. 2009;119(10):1417-23. doi:10.1161/CIRCULATIONAHA.108. 806307.

11. Hills NK, Sidney S, Fullerton HJ. Timing and number of minor infections as risk factors for childhood arterial ischemic stroke. Neurology. 2014;83(10):890-7. doi:10.1212/WNL.0000000000 000752.

12. Fink KR, Thapa MM, Ishak GE, Pruthi S. Neuroimaging of pediatric central nervous system cytomegalovirus infection. Radiographics. 2010;30(7):1779-96. doi:10.1148/rg.307105043.

13. Bale JF, Bray PF, Bell WE. Neuroradiographic abnormalities in congenital cytomegalovirus infection. Pediatr Neurol. 1984;1(1):42-7. doi:10.1016/0887-8994(85)90008-6.

14. Ancora G, Lanari M, Lazzarotto T, et al. Cranial ultrasound scanning and prediction of outcome in newborns with congenital cytomegalovirus infection. J Pediatr. 2007;150(2):157-61. doi:10.1016/j.jpeds.2006.11.032.

15. Bonthius DJ. Lymphocytic choriomeningitis virus: an underrecognized cause of neurologic disease in the fetus, child, and adult. Semin Pediatr Neurol. 2012;19(3):89-95. doi:10.1016/j.spen. 2012.02.002.

16. Briggs TA, Wolf NI, D'Arrigo S, et al. Band-like intracranial calcification with simplified gyration and polymicrogyria: a distinct "pseudo-TORCH" phenotype. Am J Med Genet Part A. 2008;146(24):3173-80. doi:10.1002/ajmg.a.32614.

17. Brown ZA, Wald A, Morrow RA, Selke S, Zeh J, Corey L. Effect of serologic status and cesarean delivery on transmission rates of herpes simplex virus from mother to infant. Obstet Gynecol Surv. 2003;58(6):376-8. doi:10.1097/01.OGX.0000070126.90718.7B.

18. Bajaj M, Mody S, Natarajan G. Clinical and neuroimaging findings in neonatal herpes simplex virus infection. J Pediatr. 2014;165(2):404-7. doi:10.1016/j.jpeds.2014.04.046.

19. Küker W, Nägele T, Schmidt F, Heckl S, Herrlinger U. Diffusion-weighted MRI in herpes simplex encephalitis: a report of three cases. Neuroradiology. 2004;46:122-5. doi:10.1007/ s00234-003-1145-3.

20. Okanishi T, Yamamoto H, Hosokawa T, et al. Diffusion-weighted MRI for early diagnosis of neonatal herpes simplex encephalitis. Brain Dev. 2015;37(4):423-31. doi:10.1016/j. braindev.2014.07.006.

21. Pelligra G, Lynch N, Miller SP, Sargent MA, Osiovich H. Brainstem involvement in neonatal herpes simplex virus type 2 encephalitis. Pediatrics. 2007;120(2):e442-6. doi:10.1542/peds. 2006-3757.

22. - Rubin EJ, Greene MF, Baden LR. Zika virus and microcephaly. $N$ Engl J Med. 2016:160210140035006. doi:10.1056/ NEJMe1601862. This study showed the severe complications of the emerging Zika virus on fetal development.

23. Wardlaw J, Dennis M. Zika virus infection and the eye. JAMA. 2015;313(23):2327-8. doi:10.1001/jama.

24. Sudre B, Danielsson N, Rakotoarivony LM, Bortel W Van, Zeller H, Jansa J. Zika virus infecton outbreak French Polynesia. 2014, February:1-12. http://ecdc.europa.eu/en/publications/Publicati ons/Zika-virus-French-Polynesia-rapid-risk-assessment.pdf.

25. Tokarz R, Firth C, Madhi SA, et al. Worldwide emergence of multiple clades of enterovirus 68. J Gen Virol. 2012;93(Pt 9):1952-8. doi:10.1099/vir.0.043935-0.

26. - Maloney JA, Mirsky DM, Messacar K, Dominguez SR, Schreiner T, Stence NV. MRI findings in children with acute flaccid paralysis and cranial nerve dysfunction occurring during the 2014 enterovirus d68 outbreak. AJNR Am J Neuroradiol. 2015;36(2):245-50. doi:10.3174/ajnr.A4188. This study 
deomstrated the imaging findings of Enterovirus D68 another emerging infection that the CDC followed very closely.

27. Summary of findings: investigation of acute flaccid myelitis in U.S. neurologic illness with limb weakness. Natl Cent Immun Respir Dis. 2015. http://www.cdc.gov/ncird/investigation/viral/ 2014-15/investigation.html.

28. Messacar K, Schreiner TL, Maloney JA, et al. A cluster of acute flaccid paralysis and cranial nerve dysfunction temporally associated with an outbreak of enterovirus D68 in children in Colorado, USA. Lancet. 2015;6736(14). doi:10.1016/S01406736(14)62457-0.

29. Greninger AL, Naccache SN, Messacar K, et al. A novel outbreak enterovirus D68 strain associated with acute flaccid myelitis cases in the USA (2012-14): a retrospective cohort study. Lancet Infect Dis. 2015;3099(15):10-2. doi:10.1016/S1473-3099(15)70093-9.

30. Lee KY, Lee Y-J, Kim TH, Cheon D-S, Nam S-O. Clinico-radiological spectrum in enterovirus 71 infection involving the central nervous system in children. $J$ Clin Neurosci. 2014;21(3):416-20. doi:10.1016/j.jocn.2013.04.032.

31. Wu T, Fan X-P, Wang W-Y, Yuan T-M. Enterovirus infections are associated with white matter damage in neonates. J Paediatr Child Health. 2014;50(10):817-22. doi:10.1111/jpc.12656.

32. Chen CY, Chang YC, Huang CC, Lui CC, Lee KW, Huang SC. Acute flaccid paralysis in infants and young children with enterovirus 71 infection: MR imaging findings and clinical correlates. AJNR Am J Neuroradiol. 2001;22(1):200-5.

33. Control D. Measles outbreak-California, december 2014 february 2015. Ann Emerg Med. 2015;66(1):82-3. doi:10.1016/j. annemergmed.2015.04.002.

34. Lee KY, Cho WH, Kim SH, Kim HD, Kim IO. Acute encephalitis associated with measles: MRI features. Neuroradiology. 2003;45(2):100-6. doi:10.1007/s00234-002-0884-x.

35. Lyon JB, Remigio C, Milligan T, Deline C. Acute necrotizing encephalopathy in a child with H1N1 influenza infection. Pediatr Radiol. 2010;40(2):200-5. doi:10.1007/s00247-009-1487-z.
36. Ito S, Shima S, Ueda A, Kawamura N, Asakura K, Mutoh T. Transient splenial lesion of the corpus callosum in H1N1 influenza virus-associated encephalitis/encephalopathy. Intern Med. 2011;50(8):915-8. doi:10.2169/internalmedicine.50.4147.

37. Mallewa M, Wilmshurst JM. Overview of the effect and epidemiology of parasitic central nervous system infections in African children. Semin Pediatr Neurol. 2014;21(1):19-25. doi:10.1016/j.spen.2014.02.003.

38. Donald KA, Walker KG, Kilborn T, et al. HIV Encephalopathy: pediatric case series description and insights from the clinic coalface. AIDS Res Ther. 2015;12(1):2. doi:10.1186/s12981-0140042-7.

39. Smith AB, Smirniotopoulos JG, Rushing EJ. From the archives of the AFIP: central nervous system infections associated with human immunodeficiency virus infection: radiologic-pathologic correlation. Radiographics. 2008;28(7):2033-58. doi:10.1148/rg. 287085135.

40. Shaw DWW, Cohen W. Viral infections of the CNS in children : imaging features. Ajr. 1993;160:125-33. doi:10.2214/ajr.160.1. 8416608.

41. - Thompson PM, Jahanshad N. Novel neuroimaging methods to understand how hiv affects the brain. Curr HIV/AIDS Rep. 2015:289-98. doi:10.1007/s11904-015-0268-6. Interesting article in more advanced imaging of HIV on brain development.

42. Bernaerts A, Vanhoenacker FM, Parizel PM, et al. Tuberculosis of the central nervous system: overview of neuroradiological findings. Eur Radiol. 2003;13(8):1876-90. doi:10.1007/s00330002-1608-7.

43. Burrill J, Williams CJ, Bain G, Conder G, Hine AL, Misra RR. Tuberculosis: a radiologic review. Radiographics. 2007;27(5): 1255-73. doi:10.1148/rg.275065176.

44. Starkey J, Moritani T, Kirby P. MRI of CNS fungal infections: review of aspergillosis to histoplasmosis and everything in between. Clin Neuroradiol. 2014;24(3):217-30. doi:10.1007/ s00062-014-0305-7. 